\title{
Association between women's empowerment and infant and child feeding practices in sub-Saharan Africa: an analysis of Demographic and Health Surveys
}

\author{
Muzi $\mathrm{Na}^{1, *}$, Larissa Jennings ${ }^{2}$, Sameera A Talegawkar ${ }^{1}$ and Saifuddin Ahmed ${ }^{3}$ \\ ${ }^{1}$ Center for Human Nutrition, Department of International Health, Johns Hopkins Bloomberg School of Public Health, \\ 615 N. Wolfe Street, W2041, Baltimore, MD 21205, USA: ${ }^{2}$ Social and Behavioral Interventions Program, \\ Department of International Health, Johns Hopkins Bloomberg School of Public Health, Baltimore, MD, USA: \\ ${ }^{3}$ Department of Population, Family and Reproductive Health, Johns Hopkins Bloomberg School of Public Health, \\ Baltimore, MD, USA
}

Submitted 6 May 2015: Final revision received 28 July 2015: Accepted 10 August 2015: First published online 8 September 2015

\begin{abstract}
Objective: To explore the relationship between women's empowerment and WHO recommended infant and young child feeding (IYCF) practices in sub-Saharan Africa.

Design: Analysis was conducted using data from ten Demographic and Health Surveys between 2010 and 2013. Women's empowerment was assessed by nine standard items covering three dimensions: economic, socio-familial and legal empowerment. Three core IYCF practices examined were minimum dietary diversity, minimum meal frequency and minimum acceptable diet. Separate multivariable logistic regression models were applied for the IYCF practices on dimensional and overall empowerment in each country.

Setting: Benin, Burkina Faso, Ethiopia, Mali, Niger, Nigeria, Rwanda, Sierra Leone, Uganda and Zimbabwe.

Subjects: Youngest singleton children aged $6-23$ months and their mothers ( $n$ 15 153). Results: Less than $35 \%, 60 \%$ and $18 \%$ of children $6-23$ months of age met the criterion of minimum dietary diversity, minimum meal frequency and minimum acceptable diet, respectively. In general, likelihood of meeting the recommended IYCF criteria was positively associated with the economic dimension of women's empowerment. Socio-familial empowerment was negatively associated with the three feeding criteria, except in Zimbabwe. The legal dimension of empowerment did not show any clear pattern in the associations. Greater overall empowerment of women was consistently and positively associated with multiple IYCF practices in Mali, Rwanda and Sierra Leone. However, consistent negative relationships were found in Benin and Niger. Null or mixed results were observed in the remaining countries.

Conclusions: The importance of women's empowerment for IYCF practices needs to be discussed by context and by dimension of empowerment.

\author{
Keywords \\ Women's empowerment
Infant and young child feeding practices \\ Demographic and Health Surveys \\ Sub-Saharan Africa
}

Despite the global trend of improved child nutritional status, $28 \%$ of children under the age of 5 years are still stunted in low- and middle-income countries ${ }^{(1)}$. The prevalence of stunting is the highest in sub-Saharan Africa where approximately $40 \%$ of children are affected $^{(1)}$. The risk of child undernutrition increases remarkably from 3 to 24 months of age ${ }^{(2)}$. This is because it is typically a period of time when suboptimal infant and young child feeding (IYCF) is practised, including lack of exclusive breast-feeding in the first 6 months, early introduction of complementary foods, and insufficient quality and quantity of complementary foods. Although the mechanisms are complex, suboptimal child feeding practices are well recognized as one of the leading causes of child undernutrition ${ }^{(3)}$. Intervention trials have also provided strong evidence that promoting adequate complementary feeding for children aged 6-23 months is key to reducing child stunting ${ }^{(4,5)}$.

The WHO recommends that infants be exclusively breast-fed for the first 6 months and then receive nutritionally adequate and safe complementary foods with continued breast-feeding up to 2 years of age or beyond $^{(6)}$. Adherence to these optimal feeding practices in 
many low- and middle-income countries is unsatisfactory. Only one in three or one in six 6-23-month-old children in sub-Saharan Africa were fed adequately diverse or overall acceptable diets, respectively ${ }^{(7)}$.

Women's empowerment is one of the key factors mediating intra-household resources towards optimal child-care behaviours and better nutritional outcomes ${ }^{(8)}$. Initial evidence indicates that women who are more empowered are more likely provide their young children with appropriate breast-feeding ${ }^{(9,10)}$ and complementary feeding practices such as giving complementary foods at the appropriate age ${ }^{(11)}$, increasing the number of meals ${ }^{(11)}$ and feeding with nutritious foods ${ }^{(10,11)}$.

Empowerment is commonly defined as the expansion of people's ability to make strategic life choices in a context where this ability was previously denied to them, ${ }^{,(12)}$. Women's empowerment involves the ability to influence decision making in many life aspects such as economic, socio-cultural, familial and interpersonal, and legal dimensions $^{(13,14)}$. Economically empowered women are more involved in income-generating activities and in control over finances, and often have significant control over food purchases, either to their children's benefit ${ }^{(15,16)}$ or to the improvement of their own diet ${ }^{(17)}$ or nutritional status $^{(11)}$. Studies in sub-Saharan Africa and South Asia have shown that the amount of money controlled by women is positively correlated with the amount of money spent on child-centred purposes, such as health care ${ }^{(11,18)}$. Women's socio-familial empowerment is characterized by their freedom of mobility and decision making regarding interpersonal or family affairs. Socio-familial empowerment enhances the ability to acquire resources, such as information and support from friends and relatives. Women with lower socio-familial empowerment are at higher risk of being unhealthy ${ }^{(19)}$ and mentally depressed or injured ${ }^{(20)}$ as the result of limited access to reproductive health services ${ }^{(21-23)}$ and higher risk of domestic violence ${ }^{(24)}$. Poor physical and mental health may indirectly hinder provision of timely, independent and proper feeding and child care. Legal empowerment refers to one's property rights over assets, such as land and houses. Securing women's property rights is seen as a tool to promote welfare and well-being of the women themselves and their children through increased agricultural productivity, intra-household bargaining power and financial security ${ }^{(18,25,26)}$. Previous evidence has suggested a connection between women's legal empowerment and better child nutrition ${ }^{(27)}$. As a multidimensional construct, women's empowerment can be linked to IYCF practices by multiple direct and indirect pathways (Fig. 1).

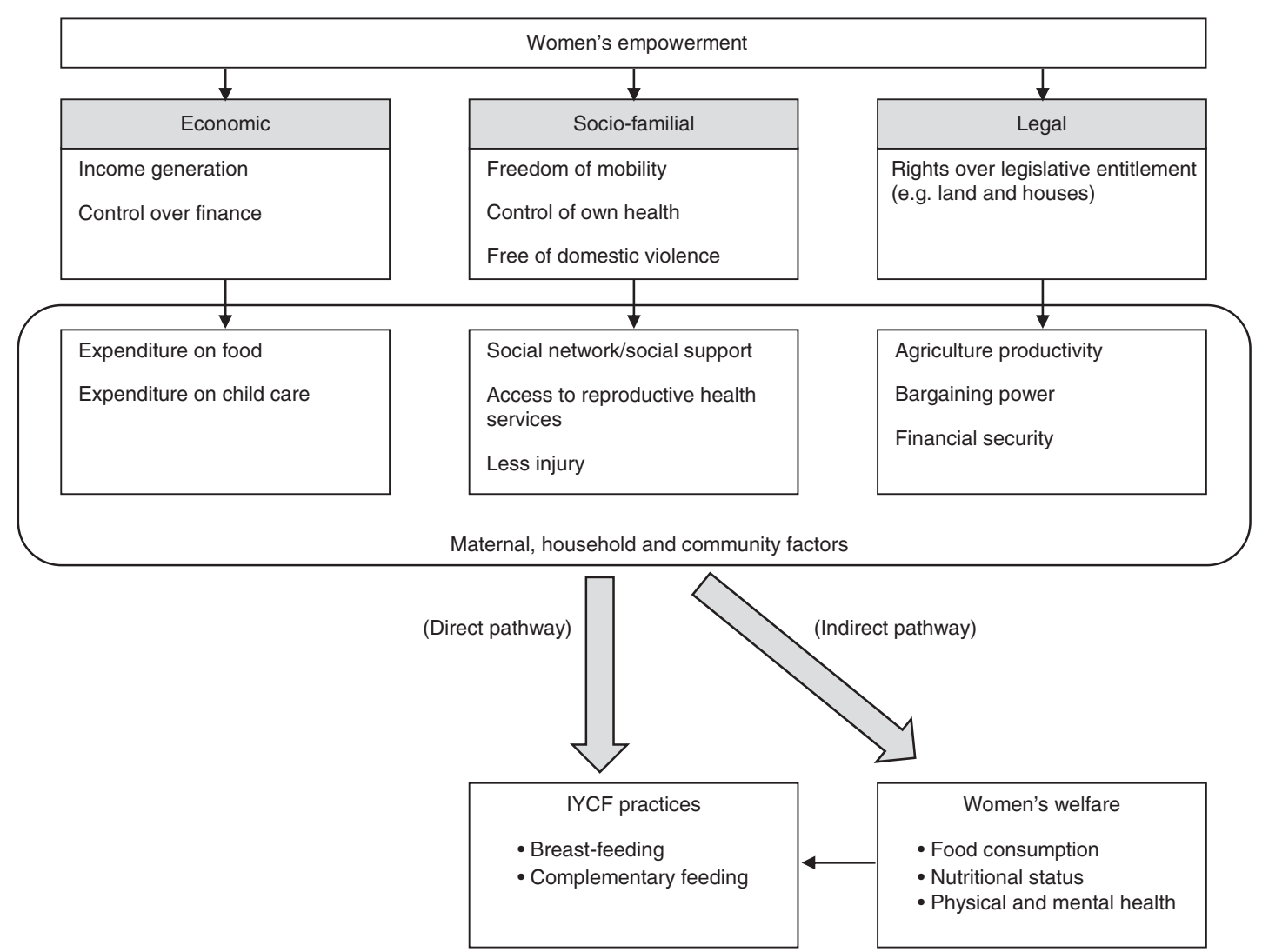

Fig. 1 Conceptual framework linking economic, socio-familial and legal women's empowerment with infant and young child feeding (IYCF) practices 
Women's empowerment may play an important role in appropriate IYCF practices. Yet the association is conditioned on many other maternal, household and community factors, such as maternal time constraints and social beliefs and norms about feeding practices ${ }^{(28)}$ (Fig. 1). For example, empowered women may enjoy more freedom to participate in the job market and to move around in their leisure time, but they may have less time available for child care and feeding ${ }^{(29-32)}$. Therefore, the direction of the association between women's empowerment and IYCF practices may be difficult to predict and may vary by context. A deeper understanding of setting-specific relationships between women's empowerment and IYCF practices is greatly needed.

Population-based evidence has suggested a positive relationship between maternal empowerment and better child nutritional status ${ }^{(33,34)}$, as well as the relationship between appropriate IYCF practices and better child nutritional outcomes ${ }^{(35,36)}$. However, little is known about how the various dimensions of women's empowerment influence IYCF practices. Therefore, the goal of the present study was to examine the association between women's empowerment status, by dimension and overall, and the uptake of WHO recommended IYCF practices in sub-Saharan African countries.

\section{Methods}

\section{Sampling}

Ten countries in sub-Saharan Africa were included in these analyses: Benin, Burkina Faso, Ethiopia, Mali, Niger, Nigeria, Rwanda, Sierra Leone, Uganda and Zimbabwe. The following inclusion criteria were used for country selection: (i) having conducted a Phase 6 Demographic and Health Survey (DHS) in the year 2010 or later; (ii) having included the women's status module with questions regarding control over income, household decision making, attitudes towards domestic violence and asset ownership; and (iii) having included the IYCF module with questions regarding breastfeeding status, complementary feeding practices and milk feeding frequency.

The sample was restricted to the youngest singleton child aged 6-23 months in the household who was alive at the time of study interview and living with the respondent, who was a married woman of reproductive age (15-49 years) living with her husband or partner at the time of survey. Following these inclusion criteria, the final sample size for each country was: $n 1908$ (Benin); $n 2090$ (Burkina Faso); $n 969$ (Ethiopia); $n 1004$ (Mali); $n 743$ (Niger); $n 4744$ (Nigeria); $n 1311$ (Rwanda); $n 1162$ (Sierra Leone); $n 849$ (Uganda); and $n 373$ (Zimbabwe).

\section{Infant and young child feeding practices}

Three core IYCF indicators for appropriate complementary feeding were created to describe adherence to the WHO guidelines: minimum dietary diversity, minimum meal frequency and minimum acceptable diet (Table 1$)^{(37)}$. Minimum dietary diversity was defined as children 6-23 months of age who received foods from at least four out of seven food groups during the past day. The seven food groups used to calculate the summed dietary diversity score were: grains, roots and tubers, legumes and nuts, flesh foods (i.e. meat, fish, poultry and organ meats), eggs, vitamin A-rich fruit and vegetables, other fruits and vegetables, and dairy products. The IYCF module in the DHS asked the respondent to report whether or not the child received each food or beverage using a pre-set list during the previous day. Positive responses were tallied by food group to create the dietary diversity score. Minimum meal frequency was defined as children 6-23 months of age who received solid, semi-solid or soft foods a minimum number of times in the previous day. The minimum required frequency varied by child age and breast-feeding status (Table 1). In the DHS, one question asked the respondent to state the number of times the

Table 1 Definition of IYCF indicators by WHO guideline ${ }^{(37)}$

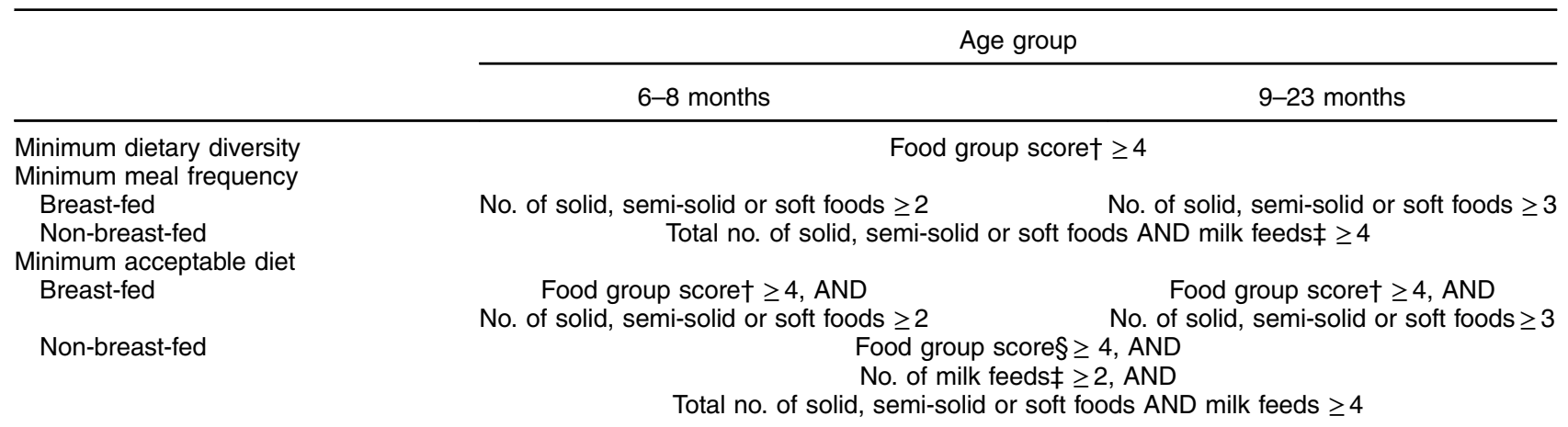

†Food group score is calculated based on consumption of seven food groups: grains, roots and tubers, legumes and nuts, dairy products, flesh foods, eggs, vitamin A-rich fruits and vegetables, and other fruits and vegetables.

$\ddagger$ Milk feeds are consumption of infant formula, milk such as tinned, powdered or fresh animal milk, and yoghurt.

$\S$ Food group score is calculated based on consumption of six food groups, excluding dairy products for avoiding double counting of this food group in nonbreast-fed children. 
child received solid, semi-solid or soft food in the past day. Three questions assessed the feeding frequency of infant formula, milk and yoghurt. These frequencies were used to calculate the number of milk feeds. Minimum acceptable diet, a combined indicator of the prior two, was defined differently for breast-fed and non-breast-fed children and for different age groups. The detailed criteria can be seen in Table 1 . The three indicators were dichotomously coded 1 if the child's feeding practice met the WHO recommendation or coded 0 if the feeding practice did not meet the recommendation.

\section{Women's empowerment variables}

In the women's status module of the DHS Phase 6 survey, nine questions captured three dimensions of women's empowerment: (i) economic empowerment (relative income to husband/partner, control over men's income, control over women's income, decision making on large household purchases); (ii) socio-familial empowerment (decision making regarding family visits, women's own health, and attitude towards domestic violence under five scenarios); and (iii) legal empowerment (women's judicial and legislative entitlements over land and over house). Following an established coding scheme ${ }^{(38)}$ we aggregated each item using dichotomous codes, with 1 indicating higher empowerment and 0 indicating lower empowerment. The summative dimensional sub-scores over items within each dimension and a total empowerment score over all nine items were calculated. The possible maximum scores for the dimensional sub-scores were 4, 3 and 2 for the economic, socio-familial and legal dimensions, respectively. The total empowerment score ranged from 0 to 9. Using DHS sample weights to adjust for probability differences in sample selection, we calculated the weighted mean and standard error of dimensional sub-scores and the total score for each country. For each empowerment dimension and total score, we categorized each woman as having either high or low empowerment based on whether her score fell above or below the country-specific weighted mean.

\section{Control variables}

Previous research has documented the association between women's empowerment and education ${ }^{(39)}$, household wealth $^{(40)}$ and number of living children ${ }^{(39,41)}$. Across studies conducted in sub-Saharan Africa, poorer maternal education, lower household wealth and younger child age have been found to be determinants of suboptimal IYCF practices $^{(42-44)}$. Given this, our analyses controlled for maternal age, maternal education, child age, child sex, number of living children, rural or urban residence and household wealth quintile as potential confounders.

\section{Statistical analysis}

The statistical software package STATA/SE version 13.1 was used to analyse data in the present study. All analyses were adjusted for complex survey design weight and design effect $>1$ due to the multistage stratified cluster sampling design of the DHS. Sample demographic characteristics, distribution of women's empowerment and distribution of IYCF practices were all described adjusting for the complex survey design to represent the population proportion, mean and standard error. To study the association between women's empowerment and IYCF practices, we applied bivariate and multivariable logistic regression models for each of the three IYCF practices by total empowerment status. Three binary dimensional empowerment variables were fitted in the unadjusted and adjusted models controlling for each other to examine the independent dimensional relationship with IYCF practices. The statistical significance level was set at $P<0 \cdot 05$.

\section{Results}

\section{Demographic characteristics and empowerment characteristics}

The sample demographic characteristics are presented by country at maternal, child and household levels (Table 2). The mean age of women ranged from $27 \cdot 8$ to $30 \cdot 3$ years across countries. Maternal education levels varied largely: the proportion of women receiving at least primary education was lowest in Burkina Faso (16.6\%) and highest in Zimbabwe $(98.6 \%)$. Children on average were between 13.4 and 14.5 months of age, with a majority of them still being breast-fed $(74 \cdot 2-93 \cdot 6 \%)$. The study sample was predominantly rural in all countries (60.4-90.0\%). However, the wealth quintile within each country sample was not always evenly distributed. For example, about half of the sample in Ethiopia (47.1\%) and Sierra Leone $(47.9 \%)$ came from the poorest two quintiles, whereas more than half of the Niger sample $(55.0 \%)$ was from the richest two quintiles.

The distribution of women's empowerment status is presented by dimensional sub-score and the total empowerment score for each country (Table 3). Across countries, the mean dimensional sub-score ranges were respectively $0 \cdot 9-3 \cdot 0,0 \cdot 6-2 \cdot 3$ and $0 \cdot 4-1 \cdot 6$ for economic, socio-familial and legal empowerment. The mean total empowerment score was lowest in Mali (2.8 (SE 0.07)) and highest in Zimbabwe (6.4 (se 0.09)).

\section{Minimum dietary diversity}

The mean dietary diversity score ranged from 1.4 in Burkina Faso to $2 \cdot 7$ in Benin and Rwanda (Table 4). Less than $35 \%$ of children 6-23 months of age met the minimum dietary diversity of at least four food groups in the past day (6.4-35.3\%). As child age increased, they were more likely to be fed with minimum required dietary diversity in their diet.

The unadjusted and adjusted odds ratios (aOR) and 95\% CI are presented (Table 5 and Table 6, respectively) between women's empowerment status and the three 
Table 2 Demographic characteristics by country; data from Phase 6 Demographic and Health Surveys conducted in sub-Saharan Africa between 2010 and 2013

\begin{tabular}{|c|c|c|c|c|c|c|c|c|c|c|}
\hline & & Burkina & & & & & & Sierra & & \\
\hline & Benin & Faso & Ethiopia & Mali & Niger & Nigeria & Rwanda & Leone & Uganda & Zimbabwe \\
\hline \multicolumn{11}{|l|}{ Maternal characteristics } \\
\hline \multicolumn{11}{|l|}{ Age (years) } \\
\hline Mean & $29 \cdot 0$ & $28 \cdot 6$ & $28 \cdot 6$ & 28.5 & $30 \cdot 3$ & $29 \cdot 3$ & 29.5 & $29 \cdot 0$ & 28.4 & $27 \cdot 8$ \\
\hline SE & 0.20 & 0.20 & 0.30 & 0.20 & 0.30 & 0.10 & 0.20 & 0.20 & 0.20 & 0.30 \\
\hline Primary education or higher (\%) & 28.5 & $16 \cdot 6$ & 33.2 & $17 \cdot 4$ & $22 \cdot 4$ & $53 \cdot 2$ & 83.7 & $28 \cdot 4$ & $87 \cdot 6$ & $98 \cdot 6$ \\
\hline \multicolumn{11}{|c|}{ Child characteristics } \\
\hline \multicolumn{11}{|l|}{ Age (months) } \\
\hline Mean & 14.5 & $14 \cdot 3$ & $14 \cdot 3$ & $14 \cdot 1$ & 13.5 & 13.8 & $14 \cdot 2$ & 13.4 & $14 \cdot 0$ & 13.5 \\
\hline SE & 0.10 & 0.10 & 0.30 & 0.20 & 0.20 & 0.10 & 0.20 & 0.20 & 0.20 & 0.30 \\
\hline Boys (\%) & $52 \cdot 4$ & $52 \cdot 2$ & $49 \cdot 1$ & $51 \cdot 2$ & 48.5 & $52 \cdot 1$ & $49 \cdot 0$ & $46 \cdot 3$ & $49 \cdot 2$ & $46 \cdot 8$ \\
\hline Still breast-fed (\%) & $76 \cdot 6$ & 93.6 & 93.0 & $88 \cdot 1$ & 86.9 & $74 \cdot 2$ & $93 \cdot 1$ & 83.7 & $80 \cdot 1$ & $80 \cdot 0$ \\
\hline \multicolumn{11}{|l|}{ Household characteristics } \\
\hline \multicolumn{11}{|l|}{ Number of living children } \\
\hline Mean & $3 \cdot 3$ & 3.4 & 3.6 & 3.7 & 4.5 & 3.6 & $3 \cdot 1$ & 3.3 & 3.9 & $2 \cdot 7$ \\
\hline SE & 0.04 & 0.05 & 0.10 & 0.07 & 0.10 & 0.04 & 0.05 & 0.06 & 0.09 & 0.08 \\
\hline Rural residence (\%) & $60 \cdot 4$ & $82 \cdot 4$ & 87.5 & 79.9 & $79 \cdot 4$ & 62.5 & $90 \cdot 0$ & $79 \cdot 6$ & 89.6 & $68 \cdot 8$ \\
\hline \multicolumn{11}{|l|}{ Wealth index quintile (\%) } \\
\hline Poorest & $17 \cdot 1$ & $21 \cdot 1$ & 23.8 & $17 \cdot 7$ & $10 \cdot 9$ & $21 \cdot 7$ & $21 \cdot 0$ & 24.7 & 17.5 & $22 \cdot 4$ \\
\hline Poorer & $19 \cdot 0$ & $20 \cdot 3$ & $23 \cdot 3$ & $19 \cdot 1$ & $14 \cdot 0$ & $21 \cdot 7$ & $22 \cdot 3$ & $23 \cdot 2$ & $25 \cdot 7$ & $17 \cdot 1$ \\
\hline Middle & 20.9 & 21.9 & $21 \cdot 1$ & $18 \cdot 8$ & $20 \cdot 1$ & 17.5 & $20 \cdot 7$ & $23 \cdot 0$ & 21.4 & 21.5 \\
\hline Richer & $21 \cdot 7$ & $22 \cdot 0$ & $16 \cdot 6$ & $26 \cdot 3$ & $26 \cdot 7$ & $19 \cdot 2$ & $20 \cdot 7$ & $16 \cdot 1$ & $20 \cdot 0$ & $22 \cdot 1$ \\
\hline Richest & $21 \cdot 2$ & $14 \cdot 7$ & $15 \cdot 2$ & $18 \cdot 0$ & $28 \cdot 3$ & $19 \cdot 8$ & $15 \cdot 3$ & 12.9 & $15 \cdot 3$ & $17 \cdot 0$ \\
\hline
\end{tabular}

Table 3 Distribution of women's empowerment indicators by dimensional sub-score and total score in each country; data from Phase 6 Demographic and Health Surveys conducted in sub-Saharan Africa between 2010 and 2013

\begin{tabular}{|c|c|c|c|c|c|c|c|c|c|c|}
\hline & & Burkina & & & & & & Sierra & & \\
\hline & Benin & Faso & Ethiopia & Mali & Niger & Nigeria & Rwanda & Leone & Uganda & Zimbabwe \\
\hline \multicolumn{11}{|l|}{ Economic } \\
\hline \multicolumn{11}{|c|}{ Dimensional sub-score (out of 4 points) } \\
\hline Mean & 1.9 & 0.9 & $2 \cdot 3$ & $1 \cdot 1$ & 1.5 & 1.6 & $2 \cdot 3$ & 1.5 & 1.9 & 3.0 \\
\hline SE & 0.03 & 0.03 & 0.05 & 0.04 & 0.04 & 0.03 & 0.04 & 0.05 & 0.05 & 0.05 \\
\hline$\%$ women > weighted mean & 63.6 & $63 \cdot 1$ & $42 \cdot 8$ & $24 \cdot 8$ & $37 \cdot 0$ & $42 \cdot 4$ & 54.6 & 53.4 & $62 \cdot 7$ & $81 \cdot 6$ \\
\hline \multirow{2}{*}{\multicolumn{11}{|c|}{$\begin{array}{l}\text { Socio-familial } \\
\text { Dimensional sub-score (out of } 3 \text { points) }\end{array}$}} \\
\hline & & & & & & & & & & \\
\hline Mean & $2 \cdot 3$ & 1.3 & 1.8 & 0.6 & 1.0 & 1.5 & 1.9 & 1.5 & 1.6 & $2 \cdot 3$ \\
\hline SE & 0.02 & 0.03 & 0.04 & 0.04 & 0.04 & 0.03 & 0.03 & 0.05 & 0.05 & 0.05 \\
\hline$\%$ women $>$ weighted mean & $49 \cdot 8$ & 39.4 & $71 \cdot 0$ & 41.5 & $30 \cdot 2$ & $45 \cdot 4$ & $74 \cdot 3$ & 55.5 & $54 \cdot 1$ & $47 \cdot 7$ \\
\hline \multirow{2}{*}{\multicolumn{11}{|c|}{$\begin{array}{l}\text { Legal } \\
\quad \text { Dimensional sub-score (out of } 2 \text { points) }\end{array}$}} \\
\hline & & & & & & & & & & \\
\hline Mean & 0.4 & 0.8 & 1.6 & $1 \cdot 1$ & 0.8 & 0.4 & 1.6 & 1.0 & $1 \cdot 1$ & $1 \cdot 1$ \\
\hline SE & 0.02 & 0.03 & 0.04 & 0.05 & 0.04 & 0.02 & 0.02 & 0.04 & 0.04 & 0.05 \\
\hline$\%$ women $>$ weighted mean & $26 \cdot 3$ & 53.5 & 74.0 & 48.8 & 53.9 & $26 \cdot 3$ & 73.9 & 59.0 & 43.5 & 42.9 \\
\hline \multicolumn{11}{|l|}{ Women's empowerment } \\
\hline \multicolumn{11}{|l|}{ Total score (out of 9 points) } \\
\hline Mean & 4.5 & $3 \cdot 0$ & $5 \cdot 7$ & $2 \cdot 8$ & $3 \cdot 3$ & 3.5 & 5.9 & $4 \cdot 0$ & $4 \cdot 7$ & $6 \cdot 4$ \\
\hline SE & 0.05 & 0.05 & 0.09 & 0.07 & 0.08 & 0.05 & 0.07 & 0.11 & $0 \cdot 10$ & 0.09 \\
\hline$\%$ women $>$ weighted mean & $57 \cdot 3$ & $58 \cdot 1$ & $61 \cdot 8$ & $54 \cdot 8$ & $38 \cdot 2$ & $42 \cdot 8$ & $66 \cdot 4$ & $60 \cdot 2$ & $53 \cdot 8$ & 51.4 \\
\hline
\end{tabular}

recommended IYCF practices by country. After controlling for confounders and the other two dimensional sub-scores (Table 6), women with higher economic empowerment were more likely to feed their children with the minimum required dietary diversity $(\mathrm{aOR}=1.05-1.43)$ as compared with women with lower economic empowerment in all countries except Benin ( $\mathrm{aOR}=0.92 ; 95 \%$ CI 0.74, 1.16), Ethiopia $(\mathrm{aOR}=0.75 ; 95 \% \mathrm{CI} 0.41,1.37)$ and Zimbabwe $(\mathrm{aOR}=0.77 ; 95 \% \mathrm{CI} 0.41,1.47)$. However, the association was significant only in Nigeria $(\mathrm{aOR}=1.40 ; 95 \%$ CI 1.15 ,
1.70). In two countries, Benin ( $\mathrm{aOR}=0 \cdot 76 ; 95 \%$ CI 0.61, $0.95)$ and Nigeria $(\mathrm{aOR}=0.80 ; 95 \%$ CI $0.66,0.98)$, there were significant negative associations between the sociofamilial dimension and the likelihood of meeting the minimum dietary diversity. Legal empowerment showed various associations across countries. While significant positive adjusted associations were found in Mali $(\mathrm{aOR}=2 \cdot 06 ; 95 \% \mathrm{CI} 1 \cdot 49,2 \cdot 85)$, Nigeria $(\mathrm{aOR}=1 \cdot 30 ; 95 \%$ CI $1 \cdot 10,1.53)$ and Sierra Leone $(\mathrm{aOR}=1.65 ; 95 \%$ CI 1.16 , $2 \cdot 35$ ), a significant negative finding was observed in Niger 
Table 4 Distribution of IYCF practices by countryt; data from Phase 6 Demographic and Health Surveys conducted in sub-Saharan Africa between 2010 and 2013

\begin{tabular}{|c|c|c|c|c|c|c|c|c|c|c|}
\hline & Benin & $\begin{array}{c}\text { Burkina } \\
\text { Faso }\end{array}$ & Ethiopia & Mali & Niger & Nigeria & Rwanda & $\begin{array}{l}\text { Sierra } \\
\text { Leone }\end{array}$ & Uganda & Zimbabwe \\
\hline \multicolumn{11}{|c|}{ Minimum dietary diversity } \\
\hline \multicolumn{11}{|c|}{ Dietary diversity score } \\
\hline Mean & $2 \cdot 7$ & 1.4 & $1 \cdot 7$ & $2 \cdot 3$ & $2 \cdot 1$ & $2 \cdot 2$ & 2.7 & $2 \cdot 0$ & $2 \cdot 5$ & $2 \cdot 6$ \\
\hline SE & 0.06 & 0.03 & 0.04 & 0.07 & 0.07 & 0.04 & 0.04 & 0.07 & 0.05 & 0.09 \\
\hline 6-11 months & $22 \cdot 7$ & 3.0 & $2 \cdot 2$ & $12 \cdot 9$ & 8.4 & $11 \cdot 6$ & $17 \cdot 8$ & $10 \cdot 7$ & $14 \cdot 7$ & $17 \cdot 3$ \\
\hline $12-17$ months & $36 \cdot 7$ & $7 \cdot 1$ & $5 \cdot 4$ & 31.5 & $16 \cdot 6$ & $23 \cdot 2$ & $30 \cdot 2$ & $19 \cdot 7$ & $26 \cdot 1$ & $36 \cdot 4$ \\
\hline 18-23 months & $46 \cdot 6$ & $9 \cdot 5$ & $9 \cdot 4$ & $37 \cdot 7$ & $26 \cdot 8$ & $27 \cdot 3$ & $31 \cdot 1$ & $25 \cdot 4$ & $27 \cdot 0$ & 37.9 \\
\hline All & $35 \cdot 3$ & $6 \cdot 4$ & $5 \cdot 6$ & $26 \cdot 8$ & $15 \cdot 9$ & $20 \cdot 2$ & $26 \cdot 1$ & $17 \cdot 7$ & $22 \cdot 1$ & $28 \cdot 8$ \\
\hline \multicolumn{11}{|c|}{ Minimum meal frequency } \\
\hline 6-11 months & $40 \cdot 9$ & $32 \cdot 5$ & $46 \cdot 5$ & $29 \cdot 3$ & $53 \cdot 2$ & 54.9 & $44 \cdot 0$ & $48 \cdot 3$ & $48 \cdot 9$ & 51.9 \\
\hline 12-17 months & $49 \cdot 8$ & 43.0 & $48 \cdot 8$ & $29 \cdot 3$ & $60 \cdot 2$ & $64 \cdot 6$ & $57 \cdot 7$ & $39 \cdot 3$ & $43 \cdot 1$ & $59 \cdot 4$ \\
\hline 18-23 months & $53 \cdot 7$ & $46 \cdot 7$ & $69 \cdot 2$ & $42 \cdot 1$ & $64 \cdot 1$ & $58 \cdot 4$ & $62 \cdot 4$ & $38 \cdot 0$ & $43 \cdot 3$ & $47 \cdot 7$ \\
\hline BF children & $48 \cdot 6$ & $40 \cdot 3$ & $53 \cdot 7$ & $31 \cdot 0$ & $59 \cdot 5$ & $62 \cdot 0$ & $54 \cdot 6$ & $46 \cdot 4$ & 44.5 & 54.9 \\
\hline Non-BF children & $46 \cdot 7$ & 43.9 & $66 \cdot 0$ & $52 \cdot 0$ & $50 \cdot 8$ & 51.9 & $51 \cdot 4$ & $20 \cdot 5$ & $48 \cdot 6$ & $47 \cdot 1$ \\
\hline All & $48 \cdot 2$ & 40.5 & $54 \cdot 5$ & 33.5 & $58 \cdot 5$ & 59.5 & $54 \cdot 4$ & $42 \cdot 4$ & $45 \cdot 3$ & 53.4 \\
\hline \multicolumn{11}{|c|}{ Minimum acceptable diet } \\
\hline 6-11 months & $16 \cdot 2$ & $2 \cdot 2$ & $2 \cdot 3$ & $8 \cdot 0$ & $5 \cdot 2$ & $9 \cdot 0$ & $12 \cdot 1$ & $5 \cdot 4$ & $6 \cdot 3$ & $12 \cdot 3$ \\
\hline $12-17$ months & $18 \cdot 9$ & 4.4 & $4 \cdot 3$ & $8 \cdot 9$ & $10 \cdot 3$ & $14 \cdot 3$ & $19 \cdot 4$ & $12 \cdot 1$ & $10 \cdot 4$ & $20 \cdot 1$ \\
\hline 18-23 months & $19 \cdot 9$ & $4 \cdot 4$ & $8 \cdot 2$ & $15 \cdot 2$ & $13 \cdot 5$ & $9 \cdot 6$ & $21 \cdot 8$ & 8.6 & $11 \cdot 7$ & $9 \cdot 8$ \\
\hline BF children & 21.5 & $3 \cdot 6$ & $5 \cdot 2$ & $10 \cdot 5$ & 9.5 & $12 \cdot 2$ & $17 \cdot 9$ & $9 \cdot 7$ & 9.4 & $16 \cdot 6$ \\
\hline Non-BF children & $7 \cdot 8$ & $4 \cdot 2$ & 0.5 & 11.4 & $6 \cdot 2$ & $8 \cdot 1$ & $13 \cdot 2$ & $3 \cdot 6$ & $8 \cdot 5$ & 3.9 \\
\hline All & $18 \cdot 3$ & 3.7 & 4.9 & $10 \cdot 6$ & $9 \cdot 1$ & $11 \cdot 2$ & $17 \cdot 6$ & $8 \cdot 8$ & $9 \cdot 2$ & 14.4 \\
\hline
\end{tabular}

IYCF, infant and young child feeding; BF, breast-fed.

†Data presented are percentages unless otherwise indicated; missing data ranges from $0 \% 10 \cdot 3 \%$.

(aOR $=0.59 ; 95 \%$ CI 0.39, 0.90). The total empowerment score had a significant and positive relationship in three countries: Burkina Faso (aOR=1.90; $95 \%$ CI 1.26, 2.88), Mali $(\mathrm{aOR}=1.54 ; 95 \% \mathrm{CI} 1.13,2.09)$ and Sierra Leone $(\mathrm{aOR}=1 \cdot 78 ; 95 \%$ CI $1 \cdot 27,2 \cdot 51)$.

\section{Minimum meal frequency}

Minimum meal frequency was achieved in 33.5-59.5\% of children (Table 4). In general, the proportion of children meeting this feeding criterion increased with child age, although a reverse trend was observed in Sierra Leone and Uganda. Data did not show any consistent trend by breastfeeding status.

Six of the ten countries demonstrated positive odds ratios $(\mathrm{aOR}=1.04-1 \cdot 29)$ in the relationship between economic empowerment and the probability of meeting the minimum meal frequency criterion (Table 6). In Burkina Faso, women with higher economic empowerment were $29 \%(\mathrm{aOR}=1.29 ; 95 \%$ CI $1.06,1.58)$ more likely to feed children at least the minimum number of meals. However, more economically empowered women in Zimbabwe were $45 \%$ less likely to provide a sufficient number of meals to their children ( $\mathrm{aOR}=0 \cdot 55 ; 95 \% \mathrm{CI} 0 \cdot 31,0 \cdot 98)$. Three significant negative findings were found in Burkina Faso $(\mathrm{aOR}=0.80 ; 95 \%$ CI 0.66, 0.98), Niger $(\mathrm{aOR}=0.57$; $95 \%$ CI $0.39,0.84)$ and Nigeria $(\mathrm{aOR}=0.80 ; 95 \% \mathrm{CI} 0.68$, 0.93 ) in relation to socio-familial empowerment. In contrast, a positive association in socio-familial empowerment and minimum meal frequency was observed in Zimbabwe $(\mathrm{aOR}=1.63 ; 95 \% \mathrm{CI} 1.01,2 \cdot 62)$. Significant negative and positive associations were observed in Benin $(\mathrm{aOR}=0.69$;
$95 \%$ CI 0.56, 0.86) and Mali (aOR=1.57; $95 \%$ CI 1.18, $2 \cdot 09$ ), respectively, between legal empowerment and minimum meal frequency. The total empowerment score was positively associated with minimum meal frequency in Mali $(\mathrm{aOR}=1.35 ; 95 \%$ CI $1.03,1.78)$ and Rwanda $(\mathrm{aOR}=1.33 ; 95 \% \mathrm{CI} 1.05,1.69)$, but negatively associated with minimum meal frequency in Benin ( $\mathrm{aOR}=0.79$; $95 \%$ CI $0.65,0.95)$ and Niger $(\mathrm{aOR}=0.64 ; 95 \% \mathrm{CI} 0.47,0.88)$ in the adjusted models.

\section{Minimum acceptable diet}

Of the children, $3 \cdot 7-18.3 \%$ were fed with minimum acceptable diet (Table 4). The likelihood of meeting this feeding criterion generally increased with child age, yet local decreases were seen between the 12-17 months and 18-23 months age group in Nigeria, Sierra Leone and Zimbabwe. No clear pattern was observable of meeting this criterion between breast-fed and non-breast-fed children.

Higher women's economic empowerment was associated with higher likelihood of feeding a minimum acceptable diet $(\mathrm{aOR}=1 \cdot 06-2 \cdot 14)$ in all countries except Ethiopia $(\mathrm{aOR}=0.67 ; 95 \%$ CI $0.33,1.36)$. Significant adjusted results were observed in Nigeria $(\mathrm{aOR}=1.43$; $95 \%$ CI $1 \cdot 11,1 \cdot 84)$ and Sierra Leone $(\mathrm{aOR}=2 \cdot 14 ; 95 \% \mathrm{CI}$ $1 \cdot 19$, 3.85; Table 6). Negative associations ( $\mathrm{aOR}=0 \cdot 54-$ 0.95) in socio-familial empowerment and minimum acceptable diet were found in six of the ten countries. However, only Nigeria showed a statistically significant negative result $(\mathrm{aOR}=0.58 ; 95 \%$ CI $0.45,0.74)$. In Zimbabwe, there was a significant positive association between women's socio-familial empowerment status and 
Table 5 Unadjusted odds ratios and $95 \% \mathrm{Cl}$ of appropriate IYCF practices among children aged 6-23 months by women's empowerment status; data from Phase 6 Demographic and Health Surveys conducted in sub-Saharan Africa between 2010 and 2013

\begin{tabular}{|c|c|c|c|c|c|c|c|c|c|c|c|c|c|c|c|c|c|c|c|c|}
\hline & \multicolumn{2}{|c|}{ Benin } & \multicolumn{2}{|c|}{ Burkina Faso } & \multicolumn{2}{|c|}{ Ethiopia } & \multicolumn{2}{|r|}{ Mali } & \multicolumn{2}{|r|}{ Niger } & \multicolumn{2}{|c|}{ Nigeria } & \multicolumn{2}{|c|}{ Rwanda } & \multicolumn{2}{|c|}{ Sierra Leone } & \multicolumn{2}{|c|}{ Uganda } & \multicolumn{2}{|c|}{ Zimbabwe } \\
\hline & OR & $95 \% \mathrm{Cl}$ & OR & $95 \% \mathrm{Cl}$ & OR & $95 \% \mathrm{Cl}$ & OR & $95 \% \mathrm{Cl}$ & OR & $95 \% \mathrm{Cl}$ & OR & $95 \% \mathrm{Cl}$ & OR & $95 \% \mathrm{Cl}$ & OR & $95 \% \mathrm{Cl}$ & OR & $95 \% \mathrm{Cl}$ & OR & $95 \% \mathrm{Cl}$ \\
\hline \multicolumn{21}{|c|}{ Minimum dietary diversity } \\
\hline Economic & 0.94 & $0.76,1.17$ & $1.80^{*}$ & $1 \cdot 15,2 \cdot 82$ & 1.31 & $0.77,2 \cdot 22$ & 1.15 & $0.82,1.62$ & $1 \cdot 18$ & $0.79,1.78$ & $1.74^{*}$ & $1 \cdot 44,2 \cdot 10$ & $1.38^{*}$ & $1.05,1.81$ & $1.49^{*}$ & $1.01,2.19$ & 1.09 & $0.75,1.60$ & 0.79 & $0.44,1.43$ \\
\hline Socio-familial & $0.80^{*}$ & $0.65,0.99$ & $1.61^{*}$ & $1.09,2.36$ & 1.33 & $0.70,2.56$ & $1.40^{*}$ & $1.03,1.90$ & 0.90 & $0.59,1.38$ & 1.06 & $0.88,1.27$ & 1.30 & $0.94,1.79$ & 1.06 & $0.72,1.56$ & 0.94 & 1.36 & 1.51 & $0.95,2.41$ \\
\hline Legal & $0.78^{*}$ & $0.63,0.97$ & 0.74 & $0.51,1.08$ & $0.45^{*}$ & $0.27,0.75$ & $1.54^{*}$ & $1 \cdot 15,2.05$ & $0.48^{*}$ & $0.33,0.70$ & $1.42^{*}$ & $1.21,1.66$ & 0.80 & $0.60,1.05$ & 1.36 & $0.99,1.87$ & $0.64^{*}$ & $0.46,0.91$ & 0.88 & $0.55,1.39$ \\
\hline Total score & 0.90 & $0.74,1.09$ & $2 \cdot 12^{*}$ & $1.41,3.17$ & 0.89 & $0.53,1.48$ & $1.43^{*}$ & $1.07,1.91$ & 0.91 & $0.63,1.31$ & $1.70^{*}$ & $1.47,1.96$ & $1.51^{*}$ & $1.15,1.98$ & $1.75^{\star}$ & $1.26,2.42$ & 0.76 & $0.55,1.06$ & 0.81 & $0.52,1.28$ \\
\hline \multicolumn{21}{|c|}{ Minimum meal frequency } \\
\hline Economic & 1.00 & $0.81,1.25$ & $1.31^{*}$ & $1.08,1.59$ & 0.94 & 0.7 & 1.05 & 0.76 & 0.96 & 0.67 & 1.06 & 1.23 & $1.27^{*}$ & 1.0 & 1.30 & 0.9 & 1.07 & 0.7 & 0.62 & 0.3 \\
\hline Socio- & 1.12 & $0.91,1.38$ & 0.83 & $0.68,1.01$ & 1.29 & 1.75 & 1.20 & 0.9 & $0.54^{*}$ & 0.77 & $0.76^{*}$ & $0.65,0.89$ & 0.99 & & 1.04 & 0.7 & 0.94 & & 1.32 & 2.02 \\
\hline Legal & $0.68^{*}$ & $0.55,0.84$ & 1.08 & $0.90,1.29$ & 0.94 & $0.70,1.24$ & $1.49^{*}$ & $1.14,1.95$ & 1.04 & $0.77,1.42$ & 0.91 & $0.79,1.04$ & $1 \cdot 18$ & 1.52 & 1.07 & 0.8 & 1.06 & 1.40 & 1.00 & $0.66,1.52$ \\
\hline Total score & $0.81^{*}$ & $0.68,0.98$ & 1.12 & $0.94,1.34$ & 1.19 & $0.91,1.55$ & 1.30 & $0.99,1.70$ & $0.64^{*}$ & $0.47,0.87$ & $0.84^{*}$ & $0.75,0.95$ & $1.46^{*}$ & $1.16,1.84$ & $1 \cdot 18$ & $0.92,1.50$ & 0.89 & $0.67,1.17$ & 0.66 & $0.44,1.00$ \\
\hline \multicolumn{21}{|c|}{ Minimum acceptable diet } \\
\hline Economic & 1.11 & $0.84,1.47$ & 1.25 & $0.73,2.14$ & 1.12 & $0.60,2.11$ & 1.24 & $0.75,2.06$ & 1.05 & $0.62,1.78$ & $1.67^{*}$ & $1 \cdot 30,2 \cdot 13$ & $1.45^{*}$ & $1.05,1.99$ & $2 \cdot 28^{\star}$ & 1.29 & $1 \cdot 18$ & 2.05 & 1.48 & $0.59,3.71$ \\
\hline Socio-familial & 0.78 & $0.60,1.03$ & $1.76^{*}$ & $1.07,2.89$ & 1.03 & $0.50,2 \cdot 12$ & 1.46 & $0.92,2.33$ & 0.68 & $0.38,1 \cdot 21$ & $0.67^{*}$ & $0.53,0.86$ & 1.21 & $0.83,1.76$ & 0.91 & $0.52,1.58$ & 1.02 & $0.60,1.72$ & $2 \cdot 31^{*}$ & $1 \cdot 22,4.37$ \\
\hline Legal & $0.67^{*}$ & $0.50,0.89$ & 0.64 & $0.40,1.04$ & 0.67 & $0.36,1.25$ & $1.84^{*}$ & $1.18,2.85$ & 0.65 & $0.40,1.05$ & 1.20 & $0.97,1.48$ & 0.81 & $0.59,1.11$ & 1.52 & $0.96,2.40$ & $0.56^{*}$ & $0.34,0.91$ & 0.99 & $0.53,1.83$ \\
\hline Total score & $0.78^{*}$ & $0.61,0.99$ & $1.97^{*}$ & $1.18,3.30$ & 1.00 & $0.54,1.87$ & 1.46 & $0.94,2.26$ & 0.61 & $0.37,1.02$ & 1.15 & $0.95,1.39$ & $1.66^{*}$ & $1 \cdot 20,2 \cdot 29$ & $1.87^{\star}$ & $1.16,3.00$ & 0.76 & $0.48,1.21$ & 1.02 & $0.56,1.87$ \\
\hline
\end{tabular}

IYCF, infant and young child feeding.

*Significant at $P<0.05$.

Table 6 Adjusted odds ratios and $95 \% \mathrm{Cl}$ of appropriate IYCF practices among children aged 6-23 months by women's empowerment statust; data from Demographic and Health Surveys conducted in sub-Saharan Africa between 2010 and 2013

\begin{tabular}{|c|c|c|c|c|c|c|c|c|c|c|c|c|c|c|c|c|c|c|c|c|}
\hline & \multicolumn{2}{|r|}{ Benin } & \multicolumn{2}{|c|}{ Burkina Faso } & \multicolumn{2}{|c|}{ Ethiopia } & \multicolumn{2}{|r|}{ Mali } & \multicolumn{2}{|r|}{ Niger } & \multicolumn{2}{|c|}{ Nigeria } & \multicolumn{2}{|c|}{ Rwanda } & \multicolumn{2}{|c|}{ Sierra Leone } & \multicolumn{2}{|c|}{ Uganda } & \multicolumn{2}{|c|}{ Zimbabwe } \\
\hline & $\mathrm{aOR}$ & $95 \% \mathrm{Cl}$ & $\mathrm{aOR}$ & $95 \% \mathrm{Cl}$ & aOR & $95 \% \mathrm{Cl}$ & $\mathrm{aOR}$ & $95 \% \mathrm{Cl}$ & aOR & $95 \% \mathrm{Cl}$ & $\mathrm{aOR}$ & $95 \% \mathrm{Cl}$ & $\mathrm{aOR}$ & $95 \% \mathrm{Cl}$ & aOR & $95 \% \mathrm{Cl}$ & aOR & $95 \% \mathrm{Cl}$ & $\mathrm{aOR}$ & $95 \% \mathrm{Cl}$ \\
\hline \multicolumn{21}{|c|}{ Minimum dietary diversity } \\
\hline Economic & 0.92 & $0.74,1.16$ & 1.43 & $0.89,2.29$ & 0.75 & $0.41,1.37$ & 1.25 & 0.87 & 1.29 & $0.82,2.03$ & $1.40^{*}$ & $1 \cdot 15,1 \cdot 70$ & 1.24 & $0.93,1.66$ & 1.35 & $0.90,2.02$ & 1.05 & 0.70 & 0.77 & $0.41,1$ \\
\hline Socio-f & $0.76^{*}$ & $0.61,0.95$ & 1.36 & $0.91,2.04$ & 0.94 & $0.46,1.91$ & 1.28 & $0.92,1.79$ & 0.71 & $0.44,1.14$ & $0.80^{*}$ & $0.66,0.98$ & 1.07 & 1.50 & 1.16 & 1.75 & 0.85 & 0.5 & 1.27 & $0.74,2.1$ \\
\hline Lega & 0.86 & $0.69,1.09$ & 0.90 & $0.61,1.34$ & 1.34 & $0.67,2.68$ & $2.06^{*}$ & $1.49,2.85$ & $0.59^{\star}$ & $0.39,0.90$ & $1.30^{*}$ & $1.10,1.53$ & 0.95 & $0.70,1.30$ & $1.65^{*}$ & $1 \cdot 16,2.35$ & 0.84 & $0.58,1.22$ & 1.83 & $0.96,3.48$ \\
\hline Total score & 0.88 & $0.72,1.07$ & $1.90^{*}$ & $1.26,2.88$ & 0.59 & $0.33,1.03$ & $1.54^{*}$ & $1.13,2.09$ & 0.84 & $0.57,1.25$ & 1.09 & $0.92,1.28$ & 1.28 & 1.72 & $1.78^{*}$ & 2.51 & 0.74 & 1.06 & 0.99 & $0.58,1.69$ \\
\hline \multicolumn{21}{|c|}{ Minimum meal frequency } \\
\hline Economic & 0.97 & $0.78,1.20$ & $1.29^{*}$ & 1.06 & 0.80 & 0.6 & 1.13 & 0.8 & 0.91 & 0.6 & 1.12 & 0.9 & 1.20 & 0. & 1.28 & 0.9 & 1.04 & 0.7 & $0.55^{*}$ & 0.31 \\
\hline Socio & 1.11 & 0.90 & $0.80^{*}$ & 0.66 & 1.17 & 0.8 & $1 \cdot 17$ & 0.87 & $0.57^{*}$ & 0.3 & $0.80^{*}$ & 93 & 0.91 & & 1.08 & & 0.96 & & $1.63^{*}$ & 1.6 \\
\hline Leg & $0.69^{*}$ & $0.56,0.86$ & 1.08 & $0.90,1.30$ & 1.09 & $0.78,1.54$ & $1.57^{\star}$ & $1.18,2.09$ & 0.96 & $0.70,1.33$ & 0.92 & 1.05 & 1.13 & 1.48 & 1.20 & & 1.18 & 0.8 & 1.52 & 0.8 \\
\hline Total score & $0.79^{\star}$ & 0.65 & 1.10 & $0.92,1.32$ & 1.06 & 0.80 & $1.35^{\star}$ & 1.03 & $0.64^{*}$ & 0.4 & 0.93 & 06 & $1.33^{*}$ & 1.69 & 1.23 & 1.59 & 0.90 & 1.20 & 0.78 & $0.50,1$. \\
\hline \multicolumn{21}{|c|}{ Minimum acceptable diet } \\
\hline Economic & 1.06 & $0.80,1.41$ & 1.14 & $0.65,2.01$ & 0.67 & $0.33,1.36$ & 1.37 & $0.81,2.31$ & 1.09 & $0.62,1.93$ & $1.43^{*}$ & $1.11,1.84$ & 1.29 & 0.9 & $2 \cdot 14^{*}$ & $1.19,3.85$ & 1.17 & 0.6 & 1.43 & $0.55,3.7$ \\
\hline nilial & 0.77 & $0.58,1.02$ & 1.62 & $0.97,2.70$ & 0.76 & $0.35,1.67$ & 1.38 & $0.85,2.24$ & 0.54 & $0.29,1.01$ & $0.58^{*}$ & $0.45,0.74$ & 1.02 & 0.6 & 0.95 & $0.54,1.69$ & 0.95 & 1.66 & $2 \cdot 27^{\star}$ & $1.11,4.64$ \\
\hline Leg & $0.72^{*}$ & $0.53,0.97$ & 0.70 & $0.43,1.15$ & 2.02 & $0.88,4.67$ & $2 \cdot 28^{\star}$ & $1.42,3.67$ & 0.79 & $0.47,1.33$ & 1.13 & $0.91,1.40$ & 0.88 & 0.6 & $1.90^{*}$ & 3.12 & 0.66 & 0.3 & $2 \cdot 18$ & $0.95,5 . c$ \\
\hline Total score & $0.76^{*}$ & $0.60,0.97$ & $1.86^{*}$ & $1 \cdot 11,3.13$ & 0.79 & $0.41,1.52$ & $1.58^{\star}$ & $1.00,2.49$ & $0.55^{\star}$ & $0.33,0.93$ & 0.88 & $0.71,1.08$ & $1.41^{*}$ & $1.01,1.98$ & $1.90^{*}$ & $1.17,3.09$ & 0.74 & $0.45,1.22$ & 1.48 & $0.75,2.6$ \\
\hline
\end{tabular}

IYCF, infant and young child feeding; aOR, adjusted odds ratio.

*Significant at $P<0.05$.

†Confounders adjusted for include maternal age, maternal education, child age, child sex, number of living children, urban residence and household wealth index quintile. 
minimum acceptable diet $(\mathrm{aOR}=2 \cdot 27 ; 95 \% \mathrm{CI} 1 \cdot 11,4 \cdot 64)$. The association between women's legal empowerment and minimum acceptable diet varied by country. In Mali $(\mathrm{aOR}=2 \cdot 28 ; 95 \% \mathrm{CI} 1.42,3.67)$ and Sierra Leone $(\mathrm{aOR}=$ 1.90; $95 \%$ CI 1·16, 3.12), legally empowered women were approximately twice as likely to feed their children with a minimum acceptable diet; while in Benin, it was about $30 \%$ less likely $(\mathrm{aOR}=0.72 ; 95 \%$ CI $0.53,0.97)$ to meet this feeding criterion. The total empowerment score was associated with increased likelihood of meeting the minimum acceptable diet in four countries: Burkina Faso $(\mathrm{aOR}=1 \cdot 86 ; 95 \% \mathrm{CI} 1 \cdot 11,3 \cdot 13)$, Mali $(\mathrm{aOR}=1 \cdot 58 ; 95 \% \mathrm{CI}$ 1.00, 2.49), Rwanda (aOR $=1.41 ; 95 \%$ CI 1.01, 1.98) and Sierra Leone $(\mathrm{aOR}=1.90 ; 95 \%$ CI 1.17, 3.09). However, the likelihood of meeting the minimum acceptable diet criterion was $24 \%(\mathrm{aOR}=0.76 ; 95 \% \mathrm{CI} 0.60,0.97)$ and $45 \%(\mathrm{aOR}=0.55 ; 95 \% \mathrm{CI} 0.33,0.93)$ less in women with higher overall empowerment in Benin and Niger, respectively.

\section{Discussion}

Using consistent measures in the DHS, we were able to explore and compare the associations between women's empowerment status and IYCF practices in ten sub-Saharan African countries. Our study showed that the strength and direction of the relationship between women's empowerment and complementary feeding practices varied by country and by dimension of empowerment.

The study's findings indicated that independent of other dimensions, economic empowerment may have positive relationships with appropriate IYCF practices, although the adjusted associations were not significant in the majority of the countries. Higher women's economic empowerment is expected to be associated with increased financial access to foods and increased food distribution to children. A study in the Philippines found that maternal contribution to household income and her control over her income were significantly related to increased weekly household food expenditure after controlling for covariates at individual and household levels ${ }^{(15)}$. Greater food expenditure may have increased women's ability to purchase food and breast milk substitutes for young children $^{(45)}$. The benefit of increased income and control over income $v$, the cost of reduced time is often recognized as a trade-off between maternal employment and child care. Existing evidence suggests that the cost is more likely to outweigh the benefit during early infancy because working mothers may not be able to initiate breastfeeding ${ }^{(29,46)}$, to practise exclusive breast-feeding ${ }^{(30)}$ or to breast-feed as long or frequently as needed ${ }^{(47-49)}$. Older children seemed to benefit more from their economically empowered mothers as seen from their better nutritional status compared with their counterparts of unemployed ${ }^{(50)}$ or less financially autonomous mothers ${ }^{(51)}$. However, women with higher financial autonomy may prioritize other urgent family demands over children's nutritional needs. In the Zimbabwean sample, where almost all women had at least primary education, we found that higher women's economic empowerment significantly decreased the probability of providing the minimum required number of meals. Maternal characteristics, such as educational attainment, may modify how household financial resources are allocated between nutritional and non-nutritional needs. More research is needed to test the potential modifying effect of socio-economic factors on the association between women's economic empowerment and IYCF practices.

Except for Zimbabwe, all statistically significant relationships between socio-familial empowerment and appropriate feeding practices were negative. A few mechanisms may help to explain the reverse direction. First, the association between socio-familial empowerment and IYCF practices may be conditioned on broader communal knowledge of IYCF practices. As women have more freedom to visit other families in the community, they may be more likely to engage in social networking with community members and exchange ideas and cultural beliefs on child-care practices. If the average local educational level is high, women may learn from each other on health and nutritional knowledge. Otherwise, they may be easily exposed to 'wrong' suggestions from the neighbourhood. In the current study, negative associations between higher socio-familial empowerment and better IYCF practices were found significant in Benin, Burkina Faso, Niger and Nigeria, where the proportion of primary education or higher was low at $28.5 \%, 16 \cdot 6 \%$, $22.4 \%$ and $53.2 \%$, respectively. However, the only positive associations of empowerment to meal frequency and to acceptable diet were observed in Zimbabwe, where maternal education was nearly universal (98.6\%). Second, the indicators under the socio-familial dimension of the current study (freedom to visit family, to decide her own health, to be against domestic violence under unjustified situations) may more closely predict behaviours that benefit women's own welfare rather than altruistic behaviours to benefit others in the family, such as children $^{(17)}$. Previous research has found close links between women's empowerment indicators that describe their own leisure and their own food consumption ${ }^{(17)}$. DHS data from thirty-one developing countries also showed that women's autonomy to decide on her own health, compared with other empowerment indicators, had the strongest relationship with better maternal healthservice utilization ${ }^{(23)}$. Because there is a potential indirect link connecting women's socio-familial empowerment, maternal sub-optimal nutritional status and health, and IYCF practices, the observed negative relationships may have been confounded by other uncontrolled factors.

In our study, both negative and positive associations were observed when examining legal empowerment. 
No consistent pattern could be captured in the relationship between legal empowerment and appropriate feeding behaviours across countries. Legal empowerment, as captured by women's legitimate ownership over land and houses, is closely associated with women's decisionmaking power in the household ${ }^{(27,52)}$ as well as protection against domestic violence ${ }^{(19,53)}$. It was not quite clear why women's title to land and houses decreased their ability to feed children appropriately in Benin and Niger compared with similar women with no land or home ownership. Because asset inheritance rules can be complicated and vary across settings ${ }^{(12)}$, a dichotomous indicator on legal empowerment may be insufficient to tell apart de facto $v$. de jure entitlement in some countries, which influences women's choice making and behaviour differently. In the context of devaluation of agriculture in India, Rao argued that granting land rights to women led to an increase of work burden without much improvements in their food security or social status ${ }^{(54)}$. Benin and Niger may have also undergone a similar shift in the valuation of land due to rapid population increase, slow growth in the agriculture sector and increased needs for diversifying livelihoods ${ }^{(54,55)}$.

Regarding overall empowerment, the direction of association was determined by the synergistic or antagonistic relationship among individual dimensional empowerment. In some countries, economic and socio-familial empowerment were negatively associated with the likelihood of appropriate feeding behaviours, which may help to explain the null relationships in overall empowerment. The insignificant associations also suggested that women's empowerment alone may not be adequate to lead to appropriate IYCF practices in many sub-Saharan countries.

Because no single indicator is sufficient to summarize appropriate complementary feeding behaviours ${ }^{(56)}$, it is important to examine multiple IYCF practices simultaneously within each country. With few exceptions, we have observed consistent significant adjusted positive (Mali, Rwanda and Sierra Leone), negative (Benin and Niger) or null associations (Ethiopia and Uganda) between women's empowerment indicators and multiple IYCF practices. The consistent direction of the associations implied correlations between IYCF practices and supported that the repeated relationships were not due to chance alone. Positive associations are in line with theoretical expectation and previous studies. In a multi-country study, pooling data from DHS 1990-1998 across thirty-six developing countries, Smith et al. observed that higher women's decision-making power relative to their partners was associated with a slight but significant increase in daily meal frequency of children aged 6-36 months and the probability of receiving complementary foods of children aged 6-12 months in South Asia and sub-Saharan Africa ${ }^{(11)}$.

The lack of significant findings in Ethiopia and Uganda may be due to several factors. Financial constraint was reported in formative research by Ethiopian ${ }^{(57)}$ and Ugandan mothers ${ }^{(58)}$ as one of the major reasons not to feed children more meals with nutritious foods. About half of the Ethiopian sample was in the two lowest wealth index quintiles, which may help explain the null average relationship. In addition, beliefs, attitudes and expectation about appropriate IYCF practices were found to deviate widely from the current guidelines ${ }^{(57,59)}$. For example, some nutrient-dense foods, such as meat and eggs, were considered unsuitable, 'too strong' or too hard to digest for young children. In Uganda, knowledge-practice discrepancies were found; mothers knew which complementary foods would be beneficial but lacked resources to put their knowledge into practice ${ }^{(60)}$. Constrained by their resources, knowledge or both, even empowered women may not be able to adhere to recommended feeding practices. In both countries, men are expected to influence feeding decisions as the major supplier of money and food ${ }^{(57,58,61)}$. Women's decisionmaking power relative to men, rather than the women's absolute empowerment, may account for more of the differences in complementary feeding practices in the two countries, yet measures on relative empowerment are not available in the DHS.

There are a few limitations inherent in the DHS data. Only associations, rather than causalities, can be studied because of the cross-sectional design of the surveys. The inclusion criteria applied in the present study required data available on multiple measurements of women's empowerment and IYCF practices. Generalizability of the study's findings may only be to subjects who share similar characteristics. Although consistent methods were used in this multi-country analysis, caution is needed when comparing results from different settings as the DHS were not conducted in the same year and not all surveys included their country-specific questions in assessing IYCF practices. Future studies are needed to examine the association in other regions of the world, such as South Asia, where women's status is low and child malnutrition is prevalent.

\section{Conclusions}

Poor complementary feeding practices to children 6-23 months of age were prevalent in the ten countries examined in sub-Saharan Africa. In general, mothers with higher economic empowerment were more likely to feed infants and young children with the minimum recommended dietary diversity and meal frequency. However, the associations in relation to socio-familial and legal empowerment need to be discussed under specific contexts as null findings and results opposite to theoretical expectations were found in several countries. The study highlighted the importance of maternal empowerment for appropriate child feeding behaviours. Future research should explore these relationships across other developing regions or study the context-specific mechanisms behind them. 


\section{Acknowledgements}

Acknowledgements: The authors acknowledge Dr Keith $\mathrm{P}$ West Jr at the Center for Human Nutrition, Department of International Health, Johns Hopkins Bloomberg School of Public Health, for his technical scope and general guidance; and Bess Lewis, MS, at Johns Hopkins Bloomberg School of Public Health for her critical comments on this manuscript. Financial support: The Bill and Melinda Gates Foundation, Seattle, WA, USA (grant number GH 614) and the Sight and Life Research Institute, Baltimore, MD, USA provided financial support for the first author. The funders had no role in the design, analysis or writing of this article. Conflict of interest: None. Authorship: M.N. conceptualized the research question, obtained data sets, reviewed literature, conducted the analysis and drafted the first draft of the manuscript. L.J., S.A.T. and S.A. provided guidance and comments at the stage of study conception, literature review, data interpretation and manuscript editing. All authors have read and approved the final version of the paper and its submission. Ethics of buman subject participation: This study was exempt as described in the guidelines issued by the Johns Hopkins Institutional Review Board.

\section{References}

1. Black RE, Victora CG, Walker SP et al. (2013) Maternal and child undernutrition and overweight in low-income and middle-income countries. Lancet 382, 427-451.

2. Victora CG, de Onis M, Hallal PC et al. (2010) Worldwide timing of growth faltering: revisiting implications for interventions. Pediatrics 125, e473-e480.

3. Black RE, Allen LH, Bhutta ZA et al. (2008) Maternal and child undernutrition: global and regional exposures and health consequences. Lancet 371, 243-260.

4. Dewey KG \& Adu-Afarwuah S (2008) Systematic review of the efficacy and effectiveness of complementary feeding interventions in developing countries. Matern Child Nutr $\mathbf{4}$, Suppl. 1, 24-85.

5. Lassi ZS, Das JK, Zahid G et al. (2013) Impact of education and provision of complementary feeding on growth and morbidity in children less than 2 years of age in developing countries: a systematic review. BMC Public Health 13, Suppl. 3, S13.

6. World Health Organization (2003) Global Strategy for Infant and Young Child Feeding. Geneva: WHO.

7. Lutter CK, Daelmans BM, de Onis M et al. (2011) Undernutrition, poor feeding practices, and low coverage of key nutrition interventions. Pediatrics 128, e1418-e1427.

8. Ruel MT, Alderman H \& Maternal and Child Nutrition Study Group (2013) Nutrition-sensitive interventions and programmes: how can they help to accelerate progress in improving maternal and child nutrition? Lancet 382, 536-551.

9. Shroff MR, Griffiths PL, Suchindran C et al. (2011) Does maternal autonomy influence feeding practices and infant growth in rural India? Soc Sci Med 73, 447-455.

10. Bose S (2011) The effect of women's status and community on the gender differential in children's nutrition in India. J Biosoc Sci 43, 513-533.

11. Smith LC, Ramakrishnan U, Ndiaye A et al. (2003) The Importance of Women's Status for Child Nutrition in
Developing Countries. Research Report no. 131. Washington, DC: International Food Policy Research Institute.

12. Kabeer N (1999) Resources, agency, achievements: reflections on the measurement of women's empowerment. Dev Change 30, 435-464.

13. Lee-Rife SM (2010) Women's empowerment and reproductive experiences over the lifecourse. Soc Sci Med $\mathbf{7 1}$, 634-642.

14. Malhotra A \& Schuler SR (2005) Women's empowerment as a variable in international development. In Measuring Empowerment: Cross-Disciplinary Perspectives, pp. 71-88 [D Narayan, editor]. Washington, DC: World Bank.

15. Schmeer KK (2005) Married women's resource position and household food expenditures in Cebu, Philippines. J Marriage Fam 67, 399-409.

16. Begin F, Frongillo EA Jr \& Delisle H (1999) Caregiver behaviors and resources influence child height-for-age in rural Chad. J Nutr 129, 680-686.

17. Basu AM \& Koolwal GB (2005) Two concepts of female empowerment: some leads from DHS data on women's status and reproductive health. In A Focus on Gender: Collected Papers on Gender Using DHS Data, pp. 15-54 [S Kishor, editor]. Calverton, MD: ORC Macro.

18. Quisumbing AR \& Maluccio JA (2003) Resources at marriage and intrahousehold allocation: evidence from Bangladesh, Ethiopia, Indonesia, and South Africa. Oxf Bull Econ Stat 65, 283-327.

19. Grown C, Gupta GR \& Pande R (2005) Taking action to improve women's health through gender equality and women's empowerment. Lancet 365, 541-543.

20. Forte JA, Franks DD, Forte JA et al. (1996) Asymmetrical role-taking: comparing battered and nonbattered women. Soc Work 41, 59-73.

21. Bloom SS, Wypij D \& Gupta MD (2001) Dimensions of women's autonomy and the influence on maternal health care utilization in a north Indian city. Demography 38, $67-78$.

22. Furuta M \& Salway S (2006) Women's position within the household as a determinant of maternal health care use in Nepal. Int Fam Plan Perspect 32, 17-27.

23. Ahmed S, Creanga AA, Gillespie DG et al. (2010) Economic status, education and empowerment: implications for maternal health service utilization in developing countries. PLoS One 5, e11190.

24. Ludermir AB, Lewis G, Valongueiro SA et al. (2010) Violence against women by their intimate partner during pregnancy and postnatal depression: a prospective cohort study. Lancet 376, 903-910.

25. Saito KA, Mekonnen H \& Spurling D (1994) Raising the Productivity of Women Farmers in Sub-Sabaran Africa. Report no. 230. Washington, DC: World Bank.

26. Datta N (2006) Joint titling - a win-win policy? Gender and property rights in urban informal settlements in Chandigarh, India. Fem Econ 12, 271-298.

27. Allendorf K (2007) Do women's land rights promote empowerment and child health in Nepal? World Dev 35 , $1975-1988$.

28. Stewart CP, Iannotti L, Dewey KG et al. (2013) Contextualising complementary feeding in a broader framework for stunting prevention. Matern Child Nutr 9, 27-45.

29. Hazir T, Akram D, Nisar YB et al. (2013) Determinants of suboptimal breast-feeding practices in Pakistan. Public Health Nutr 16, 659-672.

30. Setegn T, Belachew T, Gerbaba M et al. (2012) Factors associated with exclusive breastfeeding practices among mothers in Goba district, south east Ethiopia: a crosssectional study. Int Breastfeed J 7, 17.

31. Batal M, Boulghourian C \& Akik C (2010) Complementary feeding patterns in a developing country: a cross-sectional study across Lebanon. East Mediterr Health J 16, 180-186. 
32. Sethuraman K, Lansdown R \& Sullivan K (2006) Women's empowerment and domestic violence: the role of sociocultural determinants in maternal and child undernutrition in tribal and rural communities in south India. Food Nutr Bull 27, 128-143.

33. Carlson GJ, Kordas K \& Murray-Kolb LE (2014) Associations between women's autonomy and child nutritional status: a review of the literature. Matern Child Nutr (Epublication ahead of print version).

34. Cunningham K, Ruel M, Ferguson E et al. (2015) Women's empowerment and child nutritional status in South Asia: a synthesis of the literature. Matern Child Nutr 11, 1-19.

35. Marriott BP, White A, Hadden L et al. (2012) World Health Organization (WHO) infant and young child feeding indicators: associations with growth measures in 14 lowincome countries. Matern Child Nutr 8, 354-370.

36. Arimond M \& Ruel MT (2004) Dietary diversity is associated with child nutritional status: evidence from 11 demographic and health surveys. J Nutr 134, 2579-2585.

37. World Health Organization (2010) Indicators for Assessing Infant and Young Child Feeding Practices: Part 2 Measurement. Geneva: WHO.

38. Jennings L, Na M, Cherewick M et al. (2014) Women's empowerment and male involvement in antenatal care: analyses of Demographic and Health Surveys (DHS) in selected African countries. BMC Pregnancy Childbirth 14, 297.

39. Woldemicael G (2010) Do women with higher autonomy seek more maternal health care? Evidence from Eritrea and Ethiopia. Health Care Women Int 31, 599-620.

40. Jejeebhoy SJ (2000) Women's autonomy in rural India: its dimensions, determinants, and the influence of context. In Women's Empowerment and Demographic Processes: Moving Beyond Cairo, pp. 204-238 [H Presser and G Sen, editors]. New York: Oxford University Press.

41. Thapa DK \& Niehof A (2013) Women's autonomy and husbands' involvement in maternal health care in Nepal. Soc Sci Med 93, 1-10.

42. Issaka AI, Agho KE, Burns P et al. (2015) Determinants of inadequate complementary feeding practices among children aged 6-23 months in Ghana. Public Health Nutr 18, 669-678.

43. Aemro M, Mesele M, Birhanu Z et al. (2013) Dietary diversity and meal frequency practices among infant and young children aged 6-23 months in Ethiopia: a secondary analysis of Ethiopian Demographic and Health Survey 2011. J Nutr Metab 2013, 782931.

44. Victor R, Baines SK, Agho KE et al. (2014) Factors associated with inappropriate complementary feeding practices among children aged 6-23 months in Tanzania. Matern Child Nutr 10, 545-561.

45. Blau DM, Guilkey DK \& Popkin BM (1996) Infant health and the labor supply of mothers. J Hum Resour $\mathbf{3 1}$, 90-139.
46. Noble S (2001) Maternal employment and the initiation of breastfeeding. Acta Paediatr 90, 423-428.

47. Walker AR \& Adam FI (2000) Breast-feeding in sub-Saharan Africa: outlook for 2000. Public Health Nutr 3, 285-292.

48. Dennis C (2002) Breastfeeding initiation and duration: a 1990-2000 literature review. J Obstet Gynecol Neonatal Nurs 31, 12-32.

49. Ghosh R, Mascie-Taylor C \& Rosetta L (2006) Longitudinal study of the frequency and duration of breastfeeding in rural Bangladeshi women. Am J Hum Biol 18, 630-638.

50. Lamontagne JF, Engle PL \& Zeitlin MF (1998) Maternal employment, child care, and nutritional status of 12-18month-old children in Managua, Nicaragua. Soc Sci Med $\mathbf{4 6}$, 403-414.

51. Shroff M, Griffiths P, Adair L et al. (2009) Maternal autonomy is inversely related to child stunting in Andhra Pradesh, India. Matern Child Nutr 5, 64-74.

52. Schuler SR, Islam F \& Rottach E (2010) Women's empowerment revisited: a case study from Bangladesh. Dev Pract 20, 840-854.

53. Grabe S (2010) Promoting gender equality: the role of ideology, power, and control in the link between land ownership and violence in Nicaragua. Anal Soc Issues Public Policy 10, 146-170.

54. Rao N (2006) Land rights, gender equality and household food security: exploring the conceptual links in the case of India. Food Policy 31, 180-193.

55. Fan S, Omilola B \& Lambert M (2009) Public Spending for Agriculture in Africa: Trends and Composition. ReSAKSS Working Paper no. 28. Washington, DC: Regional Strategic Analysis and Knowledge Support System, International Food Policy Research Institute.

56. World Health Organization (2010) Indicators for Assessing Infant and Young Child Feeding Practices: Part 3 Country Profiles. Geneva: WHO.

57. Alive \& Thrive (2010) IYCF Practices, Beliefs and Influences in the SNNP Region, Ethiopia. Addis Ababa: Alive \& Thrive.

58. Mokori A (2012) Nutritional status, complementary feeding practices and feasible strategies to promote nutrition in returnee children aged 6-23 months in northern Uganda. $S$ Afr J Clin Nutr 25, 173-179.

59. Hadley C, Lindstrom D, Belachew T et al. (2008) Ethiopian adolescents' attitudes and expectations deviate from current infant and young child feeding recommendations. $J$ Adolesc Health 43, 253-259.

60. Wamani H, Astrom AN, Peterson S et al. (2005) Infant and young child feeding in western Uganda: knowledge, practices and socio-economic correlates. J Trop Pediatr 51, 356-361.

61. Engebretsen I, Moland KM, Nankunda J et al. (2010) Gendered perceptions on infant feeding in eastern Uganda: continued need for exclusive breastfeeding support. Int Breastfeed J 5, 13. 